\title{
Intracranial Arterial Calcificationes Can Reflect Cerebral Atherosclerosis Burden
}

\author{
Jeong-Min $\mathrm{Kim}^{\mathrm{a}}$ \\ Kwang-Yeol Park ${ }^{\mathrm{a}}$ \\ Jae-Han Bae ${ }^{a}$ \\ Su-Hyun Han \\ Hae-Bong Jeong ${ }^{a}$ \\ Daeun Jeong ${ }^{\mathrm{b}}$ \\ ${ }^{a}$ Department of Neurology, \\ Chung-Ang University Hospital, \\ Chung-Ang University \\ College of Medicine, Seoul, Korea \\ bepartment of Neurology and \\ Stroke Center, \\ Yeungnam University Medical Center, \\ Daegu, Korea
}

\begin{abstract}
Background and Purpose We investigated whether the intracranial arterial calcification status reflects the overall cerebral atherosclerosis burden.

Methods Patients with acute cerebral infarction who were admitted to a single university hospital stroke center and underwent brain computed tomography angiography (CTA) between May 2011 and December 2015 were included. We reviewed their demographic, clini$\mathrm{cal}$, and imaging data. Cerebral artery calcification was assessed from the cavernous portion of both internal carotid arteries, and patients were categorized into three groups according to the calcification status. The cerebral atherosclerosis score was calculated as the sum of the degree of stenosis of the major intracranial and extracranial arteries on brain CTA.

Results In total, 1,161 patients were included (age $=67 \pm 13$ years, mean \pm standard deviation), of which 517 were female. Intracranial arterial calcification and atherosclerosis were detected in 921 patients. The cerebral atherosclerosis score tended to increase with the calcification status (no calcification $=2.0 \pm 3.0$, mild $=3.8 \pm 3.8$, severe $=6.5 \pm 4.8 ; p<0.001$ in analysis of variance followed by the Bonferroni test). Multivariable logistic regression analysis including age, sex, vascular risk factors, body mass index, estimated glomerular filtration rate, high-sensitivity C-reactive protein, and calcification status showed that intracranial calcification was independently associated with an advanced cerebral atherosclerosis burden in a dose-dependent manner (compared to no calcification: odds ratio $=2.0$ and $95 \%$ confidence interval $=$ 1.1-3.4 for mild calcification, and odds ratio $=4.7$ and $95 \%$ confidence interval $=2.7-8.3$ for severe calcification).
\end{abstract}

Conclusions This study found that the calcification status of the cavernous portion of an internal carotid artery can reflect the overall cerebral atherosclerosis burden.

Key Words atherosclerosis, vascular calcification, cerebral infarction.
Received April 13,2018

Revised July 17, 2018

Accepted July 19, 2018

\section{Correspondence}

Kwang-Yeol Park, MD, PhD

Department of Neurology,

Chung-Ang University Hospital,

Chung-Ang University

College of Medicine,

102 Heukseok-ro, Dongjak-gu,

Seoul 06973, Korea

Tel $+82-2-6299-1502$

Fax +82-2-6299-1493

E-mail kwangyeol.park@gmail.com

\section{INTRODUCTION}

Vascular calcification is considered an active process of atherosclerosis rather than a passive product, and it occurs in up to $90 \%$ of atherosclerotic lesions. ${ }^{1}$ The mineralization of vascular structures is a consequence of regulated processes that culminate in the organized deposition of extracellular matrix by osteoblast-like cells under the influence of several humoral factors. ${ }^{2}$ Accordingly, coronary arterial calcification is regarded as a marker of the coronary atherosclerosis burden, which can be quantified using computed tomography (CT) of the coronary artery. The coronary artery calcium score measured in Hounsfield units in coronary CT has been shown to be predictive of future cardiovascular events. ${ }^{3} \mathrm{Sim}-$ ilar associations with increases in the cardiovascular event risk and/or mortality rate have been reported for vascular calcification of the aortic wall and renal arteries. ${ }^{4-6}$

Intracranial arterial calcification is commonly found in stroke patients on brain comput-

(®) This is an Open Access article distributed under the terms of the Creative Commons Attribution Non-Commercial License (https://creativecommons.org/licenses/by-nc/4.0) which permits unrestricted non-commercial use, distribution, and reproduction in any medium, provided the original work is properly cited. 
ed tomography angiography (CTA), and is most pronounced in the cavernous portion of the internal carotid arteries (ICAs), followed by the vertebral arteries (VA). ${ }^{7}$ There have been several recent reports on the risk factors for intracranial arterial calcification and its clinical significance. ${ }^{8}$ A population-based cohort study and a hospital-based study both showed that intracranial vascular calcification was related to a higher risk of stroke independently of cardiovascular risk factors. ${ }^{9,10}$ However, in contrast to the coronary vasculature, few studies have investigated the relationship between the degree of cerebral arterial calcification and the atherosclerosis burden.

In this study we sought to determine whether the calcification status of the cavernous portion of the ICAs on brain CTA is representative of the overall cerebral atherosclerosis burden.

\section{METHODS}

\section{Patient inclusion}

Patients who were admitted to a tertiary university hospital due to acute cerebral infarction or transient ischemic attack and who had undergone brain CTA between May 2011 and December 2015 were eligible for inclusion. We excluded patients with potential cardioembolic stroke or stroke due to other determined etiology with nonatherosclerotic arterial occlusion based on the criteria of the Trial of Org 10,172 in Acute Stroke Treatment. ${ }^{11}$ We reviewed the clinical and laboratory data of the patients. The vascular risk factors in this study included hypertension, defined as the use of antihypertensive medication or blood pressure $>140 / 90 \mathrm{~mm} \mathrm{Hg}$ on repeated measurement; diabetes mellitus, defined as the use of diabetic medication or a glycated hemoglobin level $>48$ $\mathrm{mmoL} / \mathrm{moL}$ at admission; a history of coronary artery disease; and current smoking status. The estimated glomerular filtration rate (GFR) was calculated using the Modification of Diet in Renal Disease formula. This study was reviewed and approved by the Institutional Review Board of Chung-Ang University Hospital (IRB No. C2013110), and it was exempted from the need to obtain informed consent due to its retrospective design.

\section{Brain CTA protocol and evaluation of intracranial arterial calcification}

All patients underwent multidetector brain CTA using a 40channel CT device (version 2.2, Brilliance, Philips Medical System, Best, the Netherlands) with the following parameters: $120 \mathrm{kVp}, 140 \mathrm{~mA}, 0.9-\mathrm{mm}$ section thickness, $0.9-\mathrm{mm}$ slice acquisition interval, and intravenous administration of $80 \mathrm{~mL}$ of iohexol (GE Healthcare, Milwaukee, WI, USA) at a rate of $5.0 \mathrm{~mL} / \mathrm{s}$. The presence of intracranial arterial calcifi- cation was examined in both cavernous portions of the ICAs on axial and coronal CTA images as described previously. ${ }^{7}$ The patients were categorized into three groups according to calcification status: no, mild, or severe calcification. Severe calcification was defined as calcification that involved a segment at least $1 \mathrm{~cm}$ long or $\geq 50 \%$ of the vessel diameter of either cavernous portion of the distal ICAs. The patients with calcification of lower severity were designated as having mild calcification.

The degree of cerebral atherosclerosis was examined from the bilateral anterior, middle, and posterior cerebral arteries (ACA, MCA, and PCA, respectively), basilar artery (BA), the intracranial portion of the ICA and VA, and the extracranial portion of the ICA and VA. Stenosis was scored as described previously: ${ }^{12} 0$, no stenosis; $1,<50 \%$ stenosis; 2 , 50-99\% stenosis; or 3, occlusion. Vertebral artery hypoplasia was not considered as a steno-occlusive lesion. The summed scores for cerebral atherosclerosis were calculated, and then categorized into quartiles, with patients in the highest quartile being considered as having advanced cerebral atherosclerosis. Advanced intracranial atherosclerosis was defined in the same manner as the sum of the intracranial atherosclerosis burden, including the ACA, MCA, PCA, distal ICA, intracranial VA, and BA. Extracranial atherosclerosis was calculated using the proximal ICA and extracranial VA atherosclerosis scores, atherosclerosis anterior circulation was calculated using the ACA, MCA, distal ICA and proximal ICA atherosclerosis scores, and atherosclerosis of the posterior circulation was calculated using the intracranial/extracranial VA, BA, and PCA atherosclerosis scores. The advanced-atherosclerosis group for each vascular bed was defined in the same manner.

\section{Statistical analysis}

Continuous data are expressed as mean \pm standard-deviation values and were compared using analysis of variance with the post hoc Bonferroni test or $t$-test. Categorical variables are expressed as the number and percentage of patients, and they were compared using the likelihood ratio test or chi-square test.

We first compared the demographic and laboratory variables according to the calcification status of the intracranial arteries. We next divided the study population into two groups (patients with and without advanced cerebral atherosclerosis), and compared demographic and laboratory variables in a bivariate analysis. Multivariable logistic regression analysis was performed to examine whether the intracranial calcification status could independently represent the cerebral atherosclerosis burden while adjusting for variables derived from the bivariate analysis and clinical significance in a stepwise 
manner. Three models were used: model 1 adjusted for age and sex; model 2 adjusted for age, sex, hypertension, diabetes mellitus, coronary artery disease, and current smoking status; and model 3 included the variables in model 2, with additional adjustment for the laboratory variables that were statistically significant in the bivariate analysis $(p<0.05)$.

In the sensitivity analysis we examined whether intracranial arterial calcification could represent the atherosclerosis burden at a particular anatomical location. We performed adjusted analyses with the aforementioned variables for the patients with advanced atherosclerosis burdens in the intracranial, extracranial, and anterior circulations. The adjusted odds ratios (ORs) were determined with 95\% confidence intervals (CIs). The diagnostic performance characteristics of vascular calcification for cerebral atherosclerosis were calculated.

A small group of patients had severe calcification of the intracranial arteries in the absence of cerebral atherosclerosis, and they were designated as a 'pure-calcification group'. That group comprised patients with severe calcification involving a segment at least $1 \mathrm{~cm}$ long or $\geq 50 \%$ of the vessel diameter of the distal ICAs, but without any discernible stenosis of the intracranial and extracranial cerebral arteries in brain CTA. We compared between those patients and the patients without calcification or atherosclerosis in order to derived pure intracranial calcification-specific risk factors, first using bivariate analysis and then multivariable logistic regression analysis that included variables with $p<0.05$ in the bivariate analysis. All of the statistical analyses were performed using SPSS version (version 22.0, IBM Corp., Armonk, NY), with a significance cutoff of $5 \%$.

\section{RESULTS}

Among 1,955 eligible patients, 1,161 patients were finally included in this study after excluding 236 patients without brain CTA and 558 patients with a potential cardioembolic source or stroke due to other etiology. The study population was aged $67 \pm 13$ years, and included 517 female patients. The basic demographic and laboratory variables are presented according to the intracranial arterial calcification status

Table 1. Distribution of demographic and laboratory variables according to cerebral artery calcification status

\begin{tabular}{|c|c|c|c|c|}
\hline & No calcification & Mild calcification & Severe calcification & $p$ \\
\hline Number of patients & 240 & 502 & 419 & \\
\hline Age, years & $55.7 \pm 13.5$ & $67.3 \pm 10.8$ & $74.1 \pm 9.7$ & $<0.001$ \\
\hline Sex, female & $101(42.1)$ & $211(42.0)$ & $205(48.9)$ & 0.052 \\
\hline \multicolumn{5}{|l|}{ Vascular risk factors } \\
\hline Hypertension & $87(36.3)$ & $281(56.0)$ & $310(74.0)$ & $<0.001$ \\
\hline Diabetes mellitus & $36(15.0)$ & $171(34.1)$ & $172(41.1)$ & $<0.001$ \\
\hline Smoking & $83(34.6)$ & $152(30.3)$ & $90(21.5)$ & $<0.001$ \\
\hline Previous stroke & $25(10.4)$ & $91(18.1)$ & $122(29.1)$ & $<0.001$ \\
\hline Coronary artery disease & $13(5.4)$ & $26(5.2)$ & $58(13.8)$ & $<0.001$ \\
\hline Body mass index, $\mathrm{kg} / \mathrm{m}^{2}$ & $24.2 \pm 3.4$ & $23.9 \pm 3.0$ & $23.3 \pm 3.2$ & 0.002 \\
\hline \multicolumn{5}{|l|}{ Laboratory variables } \\
\hline White blood cell count, $\times 10^{9} / \mathrm{L}$ & $8.1 \pm 2.8$ & $8.5 \pm 6.3$ & $8.3 \pm 3.6$ & 0.662 \\
\hline Hemoglobin, g/dL & $14.4 \pm 2.0$ & $13.8 \pm 2.0$ & $13.3 \pm 1.9$ & $<0.001$ \\
\hline Platelets, $\times 10^{9} / \mathrm{L}$ & $236 \pm 62$ & $241 \pm 82$ & $233 \pm 74$ & 0.267 \\
\hline Fasting blood glucose, $\mathrm{mmol} / \mathrm{L}$ & $7.1 \pm 2.8$ & $7.5 \pm 2.8$ & $7.6 \pm 3.4$ & 0.105 \\
\hline Hemoglobin A1c, \% & $5.9 \pm 1.1$ & $6.3 \pm 1.5$ & $6.3 \pm 1.4$ & $<0.001$ \\
\hline Total cholesterol, mmol/L & $4.7 \pm 1.2$ & $4.6 \pm 1.2$ & $4.6 \pm 1.3$ & 0.263 \\
\hline LDL cholesterol, mmol/L & $2.8 \pm 0.9$ & $2.8 \pm 0.9$ & $2.7 \pm 1.0$ & 0.691 \\
\hline High-sensitivity C-reactive protein, $\mathrm{mg} / \mathrm{L}$ & $5.3 \pm 24.9$ & $7.5 \pm 21.1$ & $12.7 \pm 34.0$ & 0.001 \\
\hline Estimated GFR, $\mathrm{mL} / \mathrm{min} / 1.73 \mathrm{~m}^{2}$ & $90.6 \pm 32.3$ & $74.7 \pm 25.5$ & $62.7 \pm 41.1$ & $<0.001$ \\
\hline Homocysteine, $\mu \mathrm{mol} / \mathrm{L}$ & $13.8 \pm 6.5$ & $14.5 \pm 13.2$ & $14.8 \pm 5.9$ & 0.423 \\
\hline CAS & $2.0 \pm 3.0$ & $3.8 \pm 3.8$ & $6.5 \pm 4.8$ & $<0.001$ \\
\hline Intracranial CAS & $1.3 \pm 2.3$ & $2.6 \pm 3.0$ & $4.4 \pm 4.0$ & $<0.001$ \\
\hline Extracranial CAS & $0.7 \pm 1.4$ & $1.2 \pm 1.6$ & $2.1 \pm 1.8$ & $<0.001$ \\
\hline Anterior CAS & $1.1 \pm 2.1$ & $2.3 \pm 2.4$ & $3.9 \pm 3.2$ & $<0.001$ \\
\hline Posterior CAS & $0.8 \pm 1.6$ & $1.5 \pm 2.2$ & $2.6 \pm 2.8$ & $<0.001$ \\
\hline
\end{tabular}

Data are mean \pm standard-deviation or $n(\%)$ values.

CAS: cerebral atherosclerosis score, GFR: glomerular filtration rate, LDL: low-density lipoprotein. 
in Table 1. Cerebral arterial calcification was detected in 921 patients (79.3\%). The mean age as well as the proportions of vascular risk factors including hypertension, diabetes mellitus, and previous stroke history tended to increase with the calcification status. The body mass index, hemoglobin level, and estimated GFR decreased as calcification increased. Cerebral atherosclerosis was detected in 900 patients $(77.5 \%)$ and the cerebral atherosclerosis score was $4.4 \pm 4.4$ (range $=$
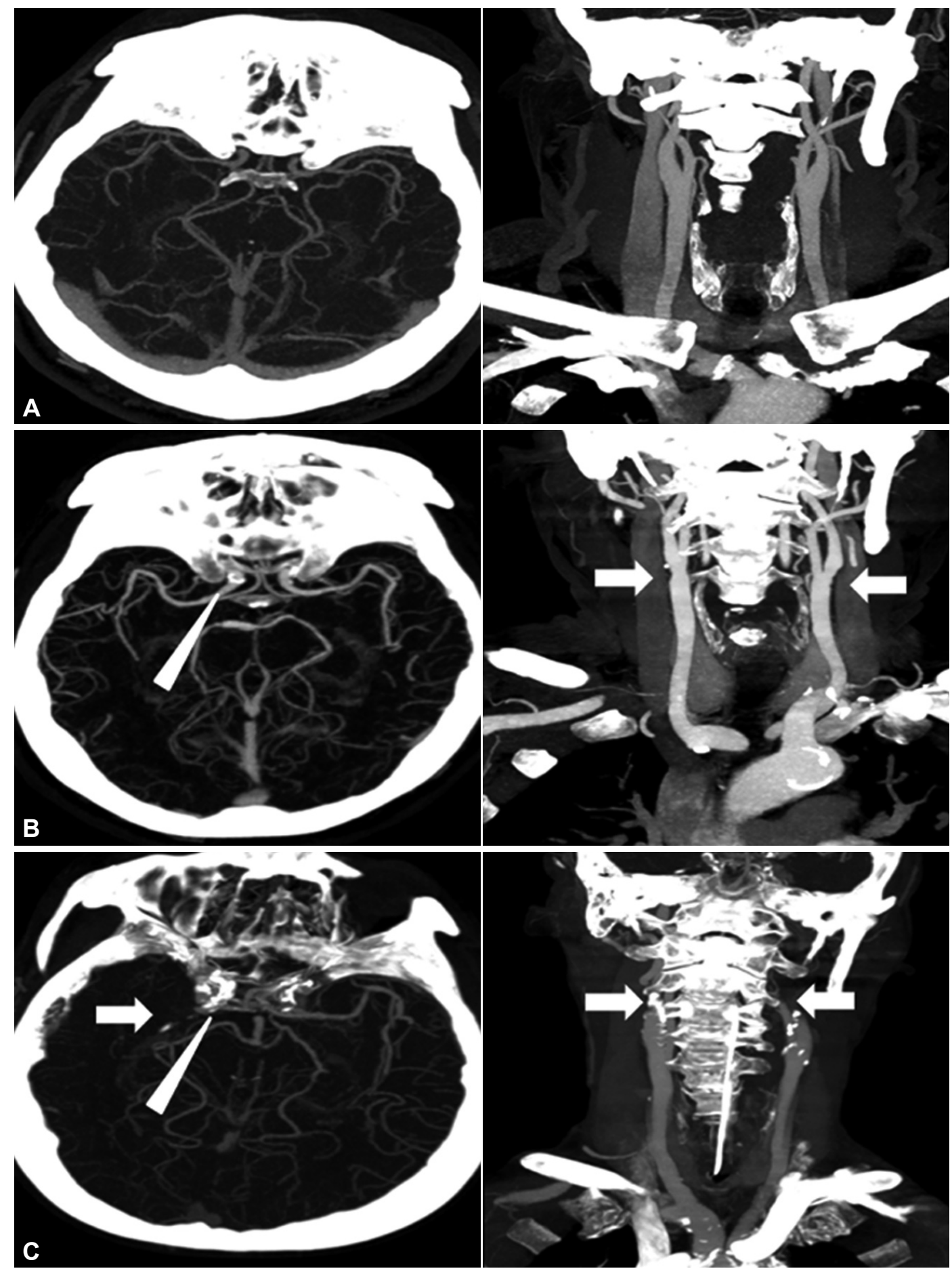

Fig. 1. Representative imaging results for cerebral arterial calcification and atherosclerosis. A: Patient A without calcification at the cavernous portion of the distal ICA had no discernible atherosclerotic burden. B: Patient B with mild calcification (arrowhead) had atherosclerotic narrowing involving both proximal ICAs (arrows). C: Patient $\mathrm{C}$ with severe intracranial arterial calcification (arrowhead) had an advanced atherosclerotic burden with occlusion of the right middle cerebral artery and left proximal ICA, and moderate stenosis of the right ICA (arrows). ICA: internal carotid artery. 
$0-25)$. The cerebral atherosclerosis scores increased with the calcification burden (no calcification $=2.0 \pm 3.0$, mild $=3.8 \pm$ 3.8 , severe $=6.5 \pm 4.8 ; p<0.001$ in analysis of variance followed by the Bonferroni test) (Table 1 and Fig. 1). When the atherosclerosis burden was considered separately according to its anatomical location, the increasing tendency of atherosclerosis was consistent across the intracranial and extracranial arteries, as well as the anterior and posterior circulation arteries (Table 1). Fig. 2 illustrates the distribution pattern of the cerebral atherosclerosis burden in terms of calcification status.

We also compared the demographic and laboratory variables of the study population according to the cerebral atherosclerosis burden. Patients with an advanced cerebral atherosclerosis burden, which was defined by a cerebral atherosclerosis score $>6$, were older at the onset and had higher proportions of hypertension, diabetes mellitus, coronary artery disease, and previous stroke history, with a low proportion of current smoking (Table 2). Their serum white blood cell count, high-

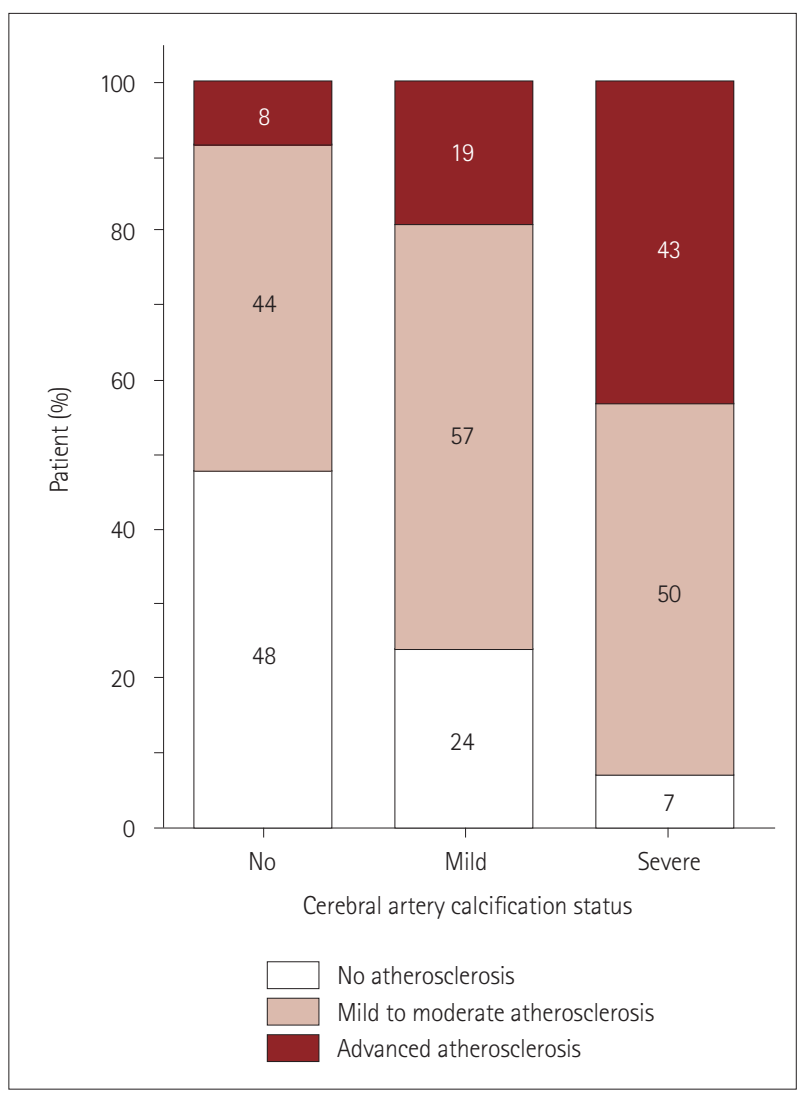

Fig. 2. Distribution of the cerebral atherosclerosis burden according to calcification status. The percentage of patients with advanced cerebral atherosclerosis tended to increase with the cerebral arterial calcification status (no calcification, 8\%; mild, 19\%; severe, 43\%; $p<0.001$ in a linear association test). Patients in the advanced cerebral atherosclerosis group were in the highest quartile range of cerebral atherosclerosis scores (>6) in brain computed tomography angiography. sensitivity C-reactive protein, and glycated hemoglobin levels were significantly higher, while the estimated GFR was significantly lower. Multivariable logistic regression analysis including age, vascular risk factors, and laboratory variables showed that the calcification status of intracranial arteries represented the advanced cerebral atherosclerosis burden in a dosedependent manner (compared to the no- calcification group: $\mathrm{OR}=2.0$ and $95 \% \mathrm{CI}=1.1-3.4$ for mild calcification, and $\mathrm{OR}=$ 4.7 and $95 \% \mathrm{CI}=2.6-8.3$ for severe calcification) (Table 3 ).

Sensitivity analyses based on the anatomical location of the atherosclerosis burden consistently demonstrated that severe cerebral artery calcification reflected advanced atherosclerosis (Table 3). The diagnostic performance measures of vascular calcification and severe vascular calcification are given in Table 4. The presence of vascular calcification showed a high sensitivity in detecting cerebral atherosclerosis (84\%) and a high specificity for detecting advanced cerebral atherosclerosis (93\%).

We observed 29 patients $(2.5 \%)$ with severe intracranial vascular calcification in the absence of cerebral atherosclerosis (pure-calcification group). Comparing these patients with the 115 patients $(9.9 \%)$ without cerebral arterial calcification or atherosclerosis revealed that they were older, had a lower

Table 2. Factors associated with advanced cerebral atherosclerosis

\begin{tabular}{|c|c|c|c|}
\hline & $\begin{array}{c}\text { Advanced } \\
\text { CAS (-) }\end{array}$ & $\begin{array}{c}\text { Advanced } \\
\text { CAS (+) }\end{array}$ & $p$ \\
\hline Number of patients & 866 & 295 & \\
\hline Age, years & $65.6 \pm 13.0$ & $72.4 \pm 11.2$ & $<0.001$ \\
\hline Sex, female & $384(44.3)$ & $133(45.1)$ & 0.83 \\
\hline \multicolumn{4}{|l|}{ Vascular risk factors } \\
\hline Hypertension & $461(53.2)$ & $217(73.6)$ & $<0.001$ \\
\hline Diabetes mellitus & $253(29.2)$ & $126(42.7)$ & $<0.001$ \\
\hline Smoking & $258(29.8)$ & $67(22.7)$ & 0.02 \\
\hline Previous stroke & $148(17.1)$ & $90(30.5)$ & $<0.001$ \\
\hline Coronary artery disease & $63(7.3)$ & $34(11.5)$ & 0.023 \\
\hline Body mass index, $\mathrm{kg} / \mathrm{m}^{2}$ & $23.8 \pm 3.1$ & $23.6 \pm 3.3$ & 0.38 \\
\hline \multicolumn{4}{|l|}{ Laboratory variables } \\
\hline White blood cell count, $\times 10^{9} / \mathrm{L}$ & $8.1 \pm 3.1$ & $9.2 \pm 7.9$ & 0.001 \\
\hline Hemoglobin, g/dL & $13.8 \pm 2.0$ & $13.6 \pm 1.9$ & 0.06 \\
\hline Platelets, $\times 10^{9} / \mathrm{L}$ & $236 \pm 73$ & $239 \pm 80$ & 0.59 \\
\hline Fasting blood sugar, mmol/L & $7.4 \pm 2.9$ & $7.8 \pm 3.4$ & 0.05 \\
\hline Hemoglobin A1c, \% & $6.1 \pm 1.3$ & $6.5 \pm 1.6$ & $<0.001$ \\
\hline Total cholesterol, mmol/L & $4.6 \pm 1.2$ & $4.6 \pm 1.4$ & 0.79 \\
\hline LDL cholesterol, mmol/L & $2.8 \pm 0.9$ & $2.7 \pm 1.0$ & 0.92 \\
\hline High-sensitivity CRP, mg/L & $7.9 \pm 25.0$ & $12.0 \pm 32.9$ & 0.03 \\
\hline Estimated GFR, $\mathrm{mL} / \mathrm{min} / 1.73 \mathrm{~m}^{2}$ & $76.5 \pm 28.9$ & $65.3 \pm 47.1$ & $<0.001$ \\
\hline Homocysteine, $\mu \mathrm{mol} / \mathrm{L}$ & $14.4 \pm 10.9$ & $14.7 \pm 5.8$ & 0.64 \\
\hline
\end{tabular}

Data are mean \pm standard-deviation or $n(\%)$ values.

CAS: cerebral atherosclerosis score, CRP: C-reactive protein, GFR: glomerular filtration rate, LDL: low-density lipoprotein. 
estimated GFR, and were more likely to have hypertension (Table 5). Multivariable logistic regression analysis of factors including age, hypertension, and estimated GFR showed that age (OR for each year $=1.1,95 \% \mathrm{CI}=1.1-1.2)$ and a history of hypertension $(\mathrm{OR}=3.1,95 \% \mathrm{CI}=1.1-8.7)$ were the independent risk factors for pure intracranial calcification without atherosclerosis.

\section{DISCUSSION}

Cerebral arterial calcification measured at the cavernous portion of the ICAs may independently represent the overall cerebral atherosclerosis burden among patients with cerebrovascular disease. The predictive values of intracranial arterial calcification in terms of the atherosclerosis burden remained significant after adjusting for clinical and laboratory variables, and throughout various vascular beds. These findings indicate that cerebral arterial calcification can be regarded as an imaging marker of cerebral atherosclerosis, in the same way that the coronary arterial calcium score represents the overall coronary atherosclerosis burden.

This study has demonstrated that intracranial arterial cal-

Table 3. Results from multivariable logistic regression analysis for predicting the advanced cerebral atherosclerosis burden

\begin{tabular}{lccc}
\hline & $\begin{array}{c}\text { Adjusting } \\
\text { model }\end{array}$ & $\begin{array}{c}\text { Mild } \\
\text { calcification }\end{array}$ & $\begin{array}{c}\text { Severe } \\
\text { calcification }\end{array}$ \\
\hline Advanced CAS & Model 1 & $2.3(1.3-4.0)$ & $6.4(3.6-11.1)$ \\
& Model 2 & $2.0(1.2-3.5)$ & $5.0(2.8-8.8)$ \\
& Model 3 & $2.0(1.1-3.4)$ & $4.7(2.6-8.3)$ \\
Vascular bed & & & \\
Advanced intracranial CAS & Model 3 & $2.6(1.5-4.6)$ & $4.8(2.7-8.8)$ \\
Advanced extracranial CAS & Model 3 & $1.5(0.9-2.5)$ & $3.0(1.7-5.0)$ \\
\hline Advanced anterior CAS & Model 3 & $1.8(1.0-3.1)$ & $4.1(2.3-7.2)$ \\
\hline
\end{tabular}

Data are odds ratio (95\% confidence interval) values. Model 1 adjusted for age and sex; model 2 adjusted for age, sex, hypertension, diabetes mellitus, coronary artery disease, and smoking status; and model 3 included the variables in model 2, with additional adjustment for highsensitivity $\mathrm{C}$-reactive protein and estimated glomerular filtration rate. CAS: cerebral atherosclerosis score. cification represents the presence of cerebral atherosclerosis with a high sensitivity and in a dose-dependent manner. The OR for the mild-calcification group to have advanced cerebral atherosclerosis was twofold higher than that for the nocalcification group, and this doubled for the severe-calcification group. Extracranial vascular calcification has generally been considered a consequence of atherosclerosis, with the exception of several specific clinical situations. ${ }^{1}$ Although the

Table 5. Factors associated with severe cerebral arterial calcification without atherosclerosis

\begin{tabular}{|c|c|c|c|}
\hline & $\begin{array}{c}\text { No } \\
\text { calcification }\end{array}$ & $\begin{array}{c}\text { Pure } \\
\text { calcification }\end{array}$ & $p$ \\
\hline Number of patients & 115 & 29 & \\
\hline Age, years & $53.9 \pm 13.2$ & $72.0 \pm 9.7$ & $<0.001$ \\
\hline Sex, female & $53(46.1)$ & $14(48.3)$ & 0.833 \\
\hline \multicolumn{4}{|l|}{ Vascular risk factors } \\
\hline Hypertension & $40(34.8)$ & $17(58.7)$ & 0.019 \\
\hline Diabetes mellitus & $19(16.5)$ & $9(31.0)$ & 0.078 \\
\hline Smoking & $43(37.4)$ & $9(31.0)$ & 0.524 \\
\hline Previous stroke & $12(10.4)$ & $6(20.7)$ & 0.204 \\
\hline Coronary artery disease & $5(4.3)$ & $3(10.3)$ & 0.201 \\
\hline Body mass index, $\mathrm{kg} / \mathrm{m}^{2}$ & $23.9 \pm 3.6$ & $23.1 \pm 3.4$ & 0.278 \\
\hline \multicolumn{4}{|l|}{ Laboratory variables } \\
\hline $\begin{array}{l}\text { White blood cell count, } \\
\times 10^{9} / \mathrm{L}\end{array}$ & $7.5 \pm 2.5$ & $9.2 \pm 4.6$ & 0.070 \\
\hline Hemoglobin, g/dL & $14.2 \pm 2.1$ & $13.8 \pm 1.9$ & 0.398 \\
\hline Platelets, $\times 10^{9} / \mathrm{L}$ & $227 \pm 57$ & $244 \pm 71$ & 0.184 \\
\hline $\begin{array}{l}\text { Fasting blood sugar, } \\
\mathrm{mmol} / \mathrm{L}\end{array}$ & $7.2 \pm 3.5$ & $7.2 \pm 4.1$ & 0.945 \\
\hline Hemoglobin A1c, \% & $5.8 \pm 1.0$ & $6.1 \pm 1.2$ & 0.082 \\
\hline Total cholesterol, $\mathrm{mmol} / \mathrm{L}$ & $4.7 \pm 1.2$ & $4.7 \pm 1.2$ & 0.824 \\
\hline LDL cholesterol, mmol/L & $2.7 \pm 0.9$ & $2.8 \pm 0.8$ & 0.616 \\
\hline High-sensitivity CRP, mg/L & $3.7 \pm 17.5$ & $17.7 \pm 46.4$ & 0.121 \\
\hline $\begin{array}{l}\text { Estimated GFR, } \\
\mathrm{mL} / \mathrm{min} / 1.73 \mathrm{~m}^{2}\end{array}$ & $92.4 \pm 30.6$ & $64.3 \pm 25.3$ & $<0.001$ \\
\hline Homocysteine, $\mu \mathrm{mol} / \mathrm{L}$ & $13.6 \pm 6.1$ & $15.6 \pm 7.9$ & 0.161 \\
\hline
\end{tabular}

Data are mean \pm standard-deviation or $n(\%)$ values.

CRP: C-reactive protein, GFR: glomerular filtration rate, LDL: low-density lipoprotein.

Table 4. Accuracy of vascular calcification in diagnosing the cerebral atherosclerosis burden

\begin{tabular}{|c|c|c|c|c|}
\hline & \multicolumn{2}{|c|}{ Calcification } & \multicolumn{2}{|c|}{ Severe calcification } \\
\hline & Atherosclerosis & Advanced CAS & Atherosclerosis & Advanced CAS \\
\hline Sensitivity, \% (95\% Cl) & $84(82-86)$ & $30(27-33)$ & 93 (90-95) & $43(38-48)$ \\
\hline Positive predictive value, \% (95\% Cl) & $86(85-88)$ & $94(91-96)$ & $43(42-45)$ & $61(56-66)$ \\
\hline Specificity, \% (95\% Cl) & $48(41-54)$ & 93 (88-95) & $31(28-35)$ & $85(82-87)$ \\
\hline Negative predictive value, $\%(95 \% \mathrm{Cl})$ & $44(39-49)$ & $26(25-27)$ & 89 (85-92) & $72(71-74)$ \\
\hline Positive likelihood ratio (95\% Cl) & $1.6(1.4-1.8)$ & $4.0(2.5-6.3)$ & $1.4(1.3-1.4)$ & $2.8(2.3-3.4)$ \\
\hline Negative likelihood ratio (95\% Cl) & $0.3(0.3-0.4)$ & $0.8(0.7-0.8)$ & $0.2(0.2-0.3)$ & $0.7(0.6-0.7)$ \\
\hline
\end{tabular}

CAS: cerebral atherosclerosis score, $\mathrm{Cl}$ : confidence interval. 
calcification of atheromatous plaques might have a stabilizing effect on the inflamed atheroma by restricting the ability of thrombogenic plaque to access the vessel lumen, its presence represents the overall atherosclerosis burden in a particular vascular bed. Accordingly, an increased burden of coronary arterial calcification predicts future cardiovascular events because it can reflect the total coronary atherosclerotic burden rather than only the stenosis of the corresponding artery. ${ }^{3}$ A previous large observational study showed that a high coronary calcium score provided independent information additional to the traditional risk factors in predicting allcause mortality in asymptomatic individuals. ${ }^{13}$ In contrast, the absence of coronary calcification was strongly associated with nonobstructive atherosclerotic lesions in coronary angiography. ${ }^{14}$ The recent Rotterdam cohort study established that intracranial atherosclerosis-as represented by the calcification volume of the cavernous ICAs-was a major risk factor for stroke in a Caucasian population. ${ }^{10}$ The relationship between calcification and stenosis in cavernous ICAs has been studied in small groups of patients receiving conventional angiography, which has shown that the presence of severe calcification is correlated with the corresponding intracranial stenosis. ${ }^{15,16}$ Our previous study showed that both intracranial arterial calcification and the overall cerebral atherosclerosis burden are associated with elevated serum homocysteine, suggesting a common pathophysiological link between these two conditions. ${ }^{12}$

Pathological studies have shown that there are four types of vascular calcification: atherosclerotic, medial arterial, cardiac valvular, and vascular calciphylaxis. ${ }^{17}$ However, these conditions are not mutually exclusive, instead frequently coexisting in clinical situations, especially atherosclerotic and medial arterial calcification. ${ }^{18}$ Atherosclerotic calcification usually occurs at sites where there is atherosclerosis of the intimal layer of the vessel wall via endochondral ossification, whereas medial vascular calcification is driven by the matrix vesicle-mediated intramembranous bone formation, which is common in diabetes, chronic kidney disease, and elderly patients. ${ }^{18}$ Since the calcification level of the vessel wall differentiates atherosclerotic and arterial medial calcification, it is impossible to differentiate these two types of calcification in the cavernous ICAs via brain CTA due to the low resolution and skull-base artifacts inherent in this imaging method. However, it might be reasonable to assume that patients with advanced calcification in the absence of cerebral atherosclerosis represent pure medial arterial calcification. A recent study investigated the correlation between the histology of the intracranial portion of the ICAs obtained from 16 autopsy patients and calcification features from brain CT, and found that the CT score based on the circularity, thickness, and continuity of intracranial ar- terial calcification can be used to differentiate between intimal and medial calcification. ${ }^{19}$ The patients in the pure-calcification group of the present study were older and more likely to have hypertension, which is consistent with the findings of previous studies. ${ }^{18}$ The presence of intracranial arterial calcification is known to be associated with increased systemic and intracranial arterial stiffness. ${ }^{20,21}$ The question of whether atherosclerotic calcification and arterial medial calcification might have distinct prognostic effects on stroke patients and therefore warrant specific treatment strategies still needs to be investigated.

The study was subject to several limitations. First, we chose to measure calcification in a semiquantitative manner via brain CTA using a previously reported rating scale. It is difficult to accurately and objectively quantify the volume of calcification from the cavernous portion of the ICAs, since the high value of Hounsfield units for the skull base is comparable to or even greater than that for vascular calcification. Another reason for using a semiquantitative method was to enable easier clinical implementation. A recent study evaluated the use of a manual quantification method in intracranial arterial beds, which might provide more-accurate information about the calcification volume in the hands of experienced clinicians. ${ }^{22}$ Second, stroke patients with known chronic kidney disease who had not undergone brain CTA studies were automatically excluded from the study, despite renal dysfunction being considered a major risk factor for vascular calcification. Third, although we excluded stroke patients with a cardioembolic source due to the difficulty of differentiating atherosclerotic occlusion from embolic occlusion, it is likely that some stroke patients with a concealed cardioembolic source were included. We similarly excluded stroke patients with nonatherosclerotic arterial occlusion, but there is a chance that some patients with moyamoya disease or arterial dissection that had not been differentiated from atherosclerosis were included in the study.

The present findings indicate that cerebral arterial calcification-with its demonstrated prognostic significance with regard to increased stroke incidence/recurrence in previous studies-appears to be a useful imaging marker for the overall cerebral atherosclerosis burden.

\section{Conflicts of Interest}

The authors have no financial conflicts of interest.

\section{Acknowledgements}

The work was supported by the Basic Science Research Program through the National Research Foundation of Korea (NRF) funded by the Ministry of Education (NRF-2016R1D1A1B03933891). The funding has no role in the design, collection, analysis, or interpretation of data; in the writing of the manuscript; and in the decision to submit the manuscript for publication. 


\section{REFERENCES}

1. New SE, Aikawa E. Cardiovascular calcification: an inflammatory disease. Circ J 2011;75:1305-1313.

2. Johnson RC, Leopold JA, Loscalzo J. Vascular calcification: pathobiological mechanisms and clinical implications. Circ Res 2006;99:10441059.

3. Polonsky TS, McClelland RL, Jorgensen NW, Bild DE, Burke GL, Guerci $\mathrm{AD}$, et al. Coronary artery calcium score and risk classification for coronary heart disease prediction. JAMA 2010;303:1610-1616.

4. Eisen A, Tenenbaum A, Koren-Morag N, Tanne D, Shemesh J, Imazio $\mathrm{M}$, et al. Calcification of the thoracic aorta as detected by spiral computed tomography among stable angina pectoris patients: association with cardiovascular events and death. Circulation 2008;118:13281334.

5. Budoff MJ, Nasir K, Katz R, Takasu J, Carr JJ, Wong ND, et al. Thoracic aortic calcification and coronary heart disease events: the multi-ethnic study of atherosclerosis (MESA). Atherosclerosis 2011;215:196-202.

6. Rifkin DE, Ix JH, Wassel CL, Criqui MH, Allison MA. Renal artery calcification and mortality among clinically asymptomatic adults. $J$ Am Coll Cardiol 2012;60:1079-1085.

7. Chung PW, Park KY, Moon HS, Kim YB, Youn YC, Byun JS, et al. Intracranial internal carotid artery calcification: a representative for cerebral artery calcification and association with white matter hyperintensities. Cerebrovasc Dis 2010;30:65-71.

8. Wu XH, Chen XY, Wang LJ, Wong KS. Intracranial artery calcification and its clinical significance. J Clin Neurol 2016;12:253-261.

9. Bugnicourt JM, Leclercq C, Chillon JM, Diouf M, Deramond H, Canaple S, et al. Presence of intracranial artery calcification is associated with mortality and vascular events in patients with ischemic stroke after hospital discharge: a cohort study. Stroke 2011;42:3447-3453.

10. Bos D, Portegies ML, van der Lugt A, Bos MJ, Koudstaal PJ, Hofman $\mathrm{A}$, et al. Intracranial carotid artery atherosclerosis and the risk of stroke in whites: the Rotterdam Study. JAMA Neurol 2014;71:405-411.

11. Adams HP Jr, Bendixen BH, Kappelle LJ, Biller J, Love BB, Gordon DL, et al. Classification of subtype of acute ischemic stroke. Definitions for use in a multicenter clinical trial. TOAST. Trial of Org 10172 in Acute Stroke Treatment. Stroke 1993;24:35-41.

12. Kim JM, Park KY, Shin DW, Park MS, Kwon OS. Relation of serum homocysteine levels to cerebral artery calcification and atherosclerosis. Atherosclerosis 2016;254:200-204.

13. Shaw LJ, Raggi P, Schisterman E, Berman DS, Callister TQ. Prognostic value of cardiac risk factors and coronary artery calcium screening for all-cause mortality. Radiology 2003;228:826-833.

14. Budoff MJ, Diamond GA, Raggi P, Arad Y, Guerci AD, Callister TQ, et al. Continuous probabilistic prediction of angiographically significant coronary artery disease using electron beam tomography. Circulation 2002;105:1791-1796.

15. Kassab MY, Gupta R, Majid A, Farooq MU, Giles BP, Johnson MD, et al. Extent of intra-arterial calcification on head CT is predictive of the degree of intracranial atherosclerosis on digital subtraction angiography. Cerebrovasc Dis 2009;28:45-48.

16. Taoka T, Iwasaki S, Nakagawa H, Sakamoto M, Fukusumi A, Takayama $\mathrm{K}$, et al. Evaluation of arteriosclerotic changes in the intracranial carotid artery using the calcium score obtained on plain cranial computed tomography scan: correlation with angiographic changes and clinical outcome. J Comput Assist Tomogr 2006;30:624-628.

17. Vattikuti R, Towler DA. Osteogenic regulation of vascular calcification: an early perspective. Am J Physiol Endocrinol Metab 2004;286:E686E696.

18. Chen NX, Moe SM. Vascular calcification: pathophysiology and risk factors. Curr Hypertens Rep 2012;14:228-237.

19. Kockelkoren R, Vos A, Van Hecke W, Vink A, Bleys RL, Verdoorn D, et al. Computed tomographic distinction of intimal and medial calcification in the intracranial internal carotid artery. PLoS One 2017;12: e0168360.

20. Park KY, Kim YB, Moon HS, Suh BC, Chung PW. Association between cerebral arterial calcification and brachial-ankle pulse wave velocity in patients with acute ischemic stroke. Eur Neurol 2009;61:364370.

21. Park KY, Chung PW, Kim YB, Moon HS, Suh BC, Yoon WT. Increased pulsatility index is associated with intracranial arterial calcification. Eur Neurol 2013;69:83-88.

22. Bos D, van der Rijk MJ, Geeraedts TE, Hofman A, Krestin GP, Witteman JC, et al. Intracranial carotid artery atherosclerosis: prevalence and risk factors in the general population. Stroke 2012;43:1878-1884. 